\title{
Experimental study of reaction of n-butanol with oxygen behind shock waves using ARAS method
}

\author{
N.S. Bystrov ${ }^{1}$, A.V. Emelianov ${ }^{1}$, A.V. Eremin ${ }^{1}$, P.I. Yatsenko ${ }^{1,2}$ \\ ${ }^{1}$ Joint Institute for High Temperatures of the Russian Academy of Sciences (JIHT RAS), Russia, Moscow, \\ 125412 Izhorskaya 13 Bldg 2 \\ ${ }^{2}$ Bauman Moscow State Technical University (BMSTU), Russia, Moscow, 105005, Mосквa, 2nd \\ Baumanskaya 5 \\ BystovNS.jiht@gmail.com
}

\begin{abstract}
This paper presents new data on the time profiles of the concentration of atomic oxygen obtained during high-temperature oxidation of n-butanol behind reflected shock waves in the temperature range of $1600-2600 \mathrm{~K}$ at pressures of 2-3 bar. The kinetics of the reaction of $\mathrm{n}$ $\mathrm{C}_{4} \mathrm{H}_{9} \mathrm{OH}$ with atomic oxygen has been studied. As a source of oxygen atoms, a small amount of nitrous oxide $\mathrm{N}_{2} \mathrm{O}$ was added to the mixture. Quantitative measurements of the concentration profiles of oxygen atoms were carried out using atomic resonance absorption spectroscopy (ARAS) on the resonance line of the $\mathrm{O}$ atom $(\lambda=130.5 \mathrm{~nm})$. Kinetic analysis of the obtained data was carried out using the Chemkin package. The experimental results obtained are compared with actual kinetic combustion schemes of n-butanol. It is shown that the kinetic schemes of n-butanol combustion available in the literature in some cases do not exactly agree with the experimental results. An analysis of possible additions to the existing kinetic schemes was carried out. As a result, it was suggested possible improvement to the existing kinetic schemes for the combustion of n-butanol at high temperatures.
\end{abstract}

Keywords: biofuel, n-butanol, ARAS, shock tube.

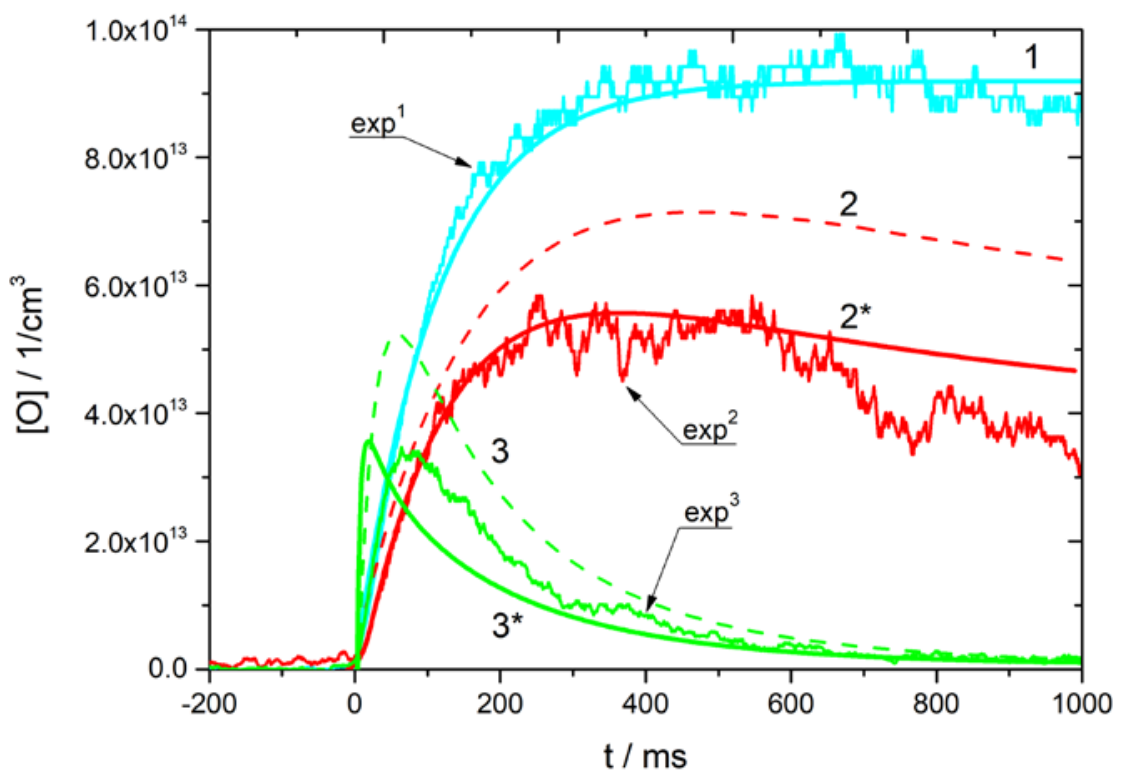

Fig. Experimental and calculated concentration profiles of $\mathrm{O}$ atoms obtained at $2100 \pm 50 \mathrm{~K}$ and $260 \pm$

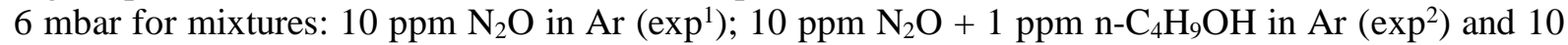
ppm $\mathrm{N}_{2} \mathrm{O}+10$ ppm n- $\mathrm{C}_{4} \mathrm{H}_{9} \mathrm{OH}$ in $\mathrm{Ar}\left(\exp ^{3}\right)$. Lines 2 (red) and 3 (green) - original kinetic mechanism of Ranzi et al., Lines $2^{*}, 3^{*}$ - Ranzi et al. [40] taking into account the reactions added to the mechanism 


\title{
Экспериментальное исследование реакции н- бутанола с кислородом за ударными волнами АРАС методом
}

\author{
Н.С. Быстров ${ }^{1}$, А.В. Емельянов ${ }^{1}$, А.В. Еремин ${ }^{1}$, П.И. Яценко ${ }^{1,2}$ \\ ${ }^{1}$ Объединённый Институт Высоких Температур Российской Академии Наук (ОИВТ РАН), \\ Россия, Москва, 125412, ул. Ижорская, д.13, стр.2 \\ ${ }^{2}$ Московский Государственный Технический Университет им. Н.Э. Баумана (МГТУ им. \\ Баумана), Россия, Москва, 105005, Москва, 2-я Бауманская ул., д. 5, стр. 1 \\ BystovNS.jiht@gmail.com
}

\begin{abstract}
Аннотация
В данной работе представлены новые данные о временных профилях концентрации атомарного кислорода, полученных при высокотемпературном окислении н-бутанола за отраженными ударными волнами в диапазоне температур 1600-2600 К при давлениях 2-3 бар. Изучена кинетика реакции $\mathrm{n}-\mathrm{C}_{4} \mathrm{H}_{9} \mathrm{OH}$ с атомарным кислородом. В качестве источников атомов кислорода в смесь добавлялось небольшое количество закиси азота $\mathrm{N}_{2} \mathrm{O}$. Количественные измерения профилей концентрации атомов кислорода проводились методом атомно-резонансной абсорбционной спектроскопии (АРАC) на резонансной линии атома $\mathrm{O}(\lambda=130.5$ нм). Кинетический анализ полученных данных проводился с использованием пакета Chemkin. Проведено сравнение полученных экспериментальных результатов с актуальными кинетическими схемами горения н-бутанола. Показано, что имеющиеся в литературе кинетические схемы горения н-бутанола в ряде случаев не совсем точно согласуются с экспериментальными результатами. Проведен анализ возможных дополнений в применяемые кинетические схемы. В результате удалось улучшить существующие кинетические схемы горения н-бутанола при высоких температурах.
\end{abstract}

Ключевые слова: биотопливо, н-бутанол, АРАС, ударная труба.

\section{1. Введение}

Множество факторов способствуют растущему во всем мире интересу к биотопливу и его свойствам горения. Ограниченность нефтяных, газовых и прочих топливных ресурсов диктует стремления к энергетической независимости практически всех стран. Истощение запасов нефти является неизбежным [1, 2], хотя новые запасы продолжают обнаруживаться. Выбросы в атмосферу парниковых газов, особенно углекислого газа, и других загрязняющих веществ в результате сжигания ископаемого топлива, представляют собой серьезную проблему глобального характера. Поэтому биотопливо предоставляет потенциал как для продления нефтяной эры (в качестве добавки к бензину/дизельному топливу), так и для её завершения (в качестве полной замены существующего топлива).

Обычное биотопливо [3] включает в себя низкомолекулярные спирты, такие как этанол, н-бутанол и изопентанол, полученные из сахара или крахмала, а также биодизельное топливо, полученное из растительных масел или жиров. В последние годы значительный интерес вызывает н-бутанол ввиду его исключительных свойств [4], потенциально, как наиболее перспективного возобновляемого экологически-чистого топлива [5]. Во многих 
странах биометанол и биоэтанол уже широко используются в качестве добавок к бензину. Теперь и н-бутанол рассматривается на данную роль, поскольку обладает некоторыми преимуществами по сравнению с более легкими спиртами, например, уже применяемым этанолом:

- $\quad$ Более энергетически выгодный - в то время как этанол имеет приблизительно две трети энергетической ценности бензина (21.2 против 32.5 МДж/л), н-бутанол имеет приблизительно 90 процентов (29.2 МДж/л).

- $\quad$ Менее летучий - н-бутанол в шесть раз менее летуч, чем этанол, выбросы которого в результате испарения вызывают смог.

- $\quad$ Менее едкий - н-бутанол не так агрессивен, потому доставляет много меньше проблем с коррозией алюминиевых и полимерных компонентов в топливных и дозирующих системах.

- Более гидрофобный - н-бутанол является более гидрофобным и может транспортироваться в существующих топливопроводах, тогда как этанол должен транспортироваться железными дорогам, баржами или грузовиками.

- $\quad$ Н-бутанол может быть использован в качестве полной замены бензина, вместо Е85 $(85 \%$ этанола $+15 \%$ бензина) и Е100 (100\% этанола), используемых в Бразилии только после значительных модификаций двигателей [4].

За последние 10-15 лет исследователями, при использовании как экспериментальных, так и теоретических подходов, тщательно изучена химия и физика горения н-бутанола [6-13]: времена задержек воспламенения [2, 3, 14], скорости ламинарных пламен [2, 3, 15-20], профили частиц, измеренных в пиролизе [21-24], горение в реактивных двигателях [25-27] и измерения в предварительно смешанных пламенах [28-30]. В результате, был предложен целый ряд кинетических моделей $[2,3,26,27,29,31]$ для описания химии горения нбутанола. Однако, большинство опубликованных экспериментальных данных о константах скорости реакций между н-бутанолом и кислородом попадают в область низких температур (300-500 К). Это означает, что необходимы дополнительные исследования при температурах, близких к реальным условиям горения и воспламенения н-бутанола, что позволит улучшить понимание кинетических закономерностей в этом процессе. Поэтому основной целью данного исследования было экспериментальное изучение взаимодействия н-бутанола с кислородом при температурах выше 1000 К.

\section{2. Эксперимент}

Все эксперименты проводились за отраженными ударными волнами в кинетической ударной трубе из нержавеющей стали круглого сечения с внутренним диаметром 108 мм. Длина камеры низкого давления составляла 6.5 м, а камеры высокого давления 2.5 м. Перед экспериментом ударная труба откачивалась безмасляным и турбомолекулярным насосами до давления $2 \cdot 10^{-7}$ мбар. Скорость натекания в ударную трубу составляла $8 \cdot 10^{-7}$ мбар/мин. Более подробное описание установки приведено в [32-35]. Используемые газы тщательно перемешивались в специальном баллоне из нержавеющей стали, который можно было нагревать для уменьшения дегазации со стенок и откачивать с помощью турбомолекулярного насоса. Аргон, используемый в качестве разбавляющего газа, был поставлен Voessen Quality Center и сертифицирован по чистоте 99.9999\%, $\mathrm{N}_{2} \mathrm{O}$ был поставлен фирмой Horst и сертифицирован по чистоте 99.9994\%, а н-бутанол (n- $\left.\mathrm{C}_{4} \mathrm{H}_{9} \mathrm{OH}\right)$ произведен ООО «Компонент-реактив» с чистотой 99.9\%.

В качестве источника излучения для метода АРАС использовалась микроволновая разрядная лампа. Для возбуждения излучения атома кислорода была приготовлена смесь $1.0 \% \mathrm{O}_{2}+$ Не. Микроволновый разряд создавался с помощью твердотельного генератора Sairem GMS-200 на частоте 2.45 ГГц. Мощность микроволнового разряда, генерируемого генератором, составляла 80 Вт. Для регистрации УФ-излучения, прошедшего через ударную трубу, 
использовались вакуумный монохроматор Acton VM-502 и фотоумножитель 181-PMT со спектральной чувствительностью в области от 112 до 830 нм. Для измерений АРАС была выбрана наиболее интенсивная резонансная линия атома О на длине волны 130.5 нм. Спектральная ширина монохроматора составляла 1.2 нм. Тестовые эксперименты на чистом аргоне, проведенные до серии экспериментов с пиролизом $\mathrm{N}_{2} \mathrm{O}$ и n- $\mathrm{C}_{4} \mathrm{H}_{9} \mathrm{OH}$, подтвердили, что поглощение в выбранном диапазоне длин волн отсутствовало.

\section{1. Калибровка}

Спектральная форма линий, излучаемых разрядной лампой, подробно не известна, и предполагается, что на нее влияет самопоглощение. Поэтому, чтобы получить достоверную связь между измеренным резонансным поглощением и соответствующими концентрациями атомов кислорода была проведена процедура калибровки. Для калибровки интенсивности поглощения атомов кислорода в зависимости от их концентрации была реализована специальная серия экспериментов в смеси, содержащей различные концентрации $\mathrm{N}_{2} \mathrm{O}$ в $\mathrm{Ar}$, при $T=2100 \pm 50 \mathrm{~K}$, когда молекула $\mathrm{N}_{2} \mathrm{O}$ полностью диссоциирует на атом $\mathrm{O}$ и молекулу $\mathrm{N}_{2}$ [36]. На рис. 1 приведены примеры профилей поглощения, полученных на длине волны 130.5 нм при температуре $2135 \pm 5 \mathrm{~K}$ и различных концентрациях $\mathrm{N}_{2} \mathrm{O}$. Хорошо виден подъем сигнала поглощения и последующий выход на стационарный уровень, что связано с образованием атомов О за отраженной ударной волной. Измеряя уровень поглощения атомов $\mathrm{O}$, и, сравнивая его со значением известной начальной концентрации кислорода (при условии полной диссоциации $\mathrm{N}_{2} \mathrm{O}$ ), была построена калибровочная кривая (рис. 2). Закон ЛамбертаБэра не совсем точно отражает реальную связь между уровнем поглощения и концентрацией (в частности, из-за различных процессов уширения и смещения испускаемых и поглощаемых линий - см., например, [37]). Поэтому обычно используется так называемый модифицированный закон Ламберта-Бэра [37]. На рис. 2 представлены экспериментальные точки, которые хорошо описываются калибровочной кривой аппроксимации, соответствующей модифицированному закону Ламберта-Бэра:

$$
A_{0}=1-\exp \left(-\sigma^{(3 \alpha-1)} \cdot l \cdot[0]^{\alpha}\right),
$$

где $A_{0}$ - поглощение атомами $\mathrm{O} ; l$ - оптический путь в см; [O] - концентрация атомов О в $\mathrm{cm}^{-3} ; \sigma=3.56 \cdot 10^{-15} \mathrm{~cm}^{2}$ - сечение поглощения на линии 130.5 нм (определенное при $\alpha=$ 1). Показатель экспоненты $\alpha$ в модифицированном законе Ламберта-Бэра варьировался до наилучшего соответствия с полученной калибровочной кривой и составил величину $=0.7$ (см. гладкую кривую на рис. 2). Аналогичные зависимости для атомов кислорода были получены в [37-38]. В дальнейших исследованиях с использованием этой калибровочной кривой текущая концентрация атомов $\mathrm{O}$ может быть точно определена в соответствии с уровнем сигнала поглощения при различных температурах.
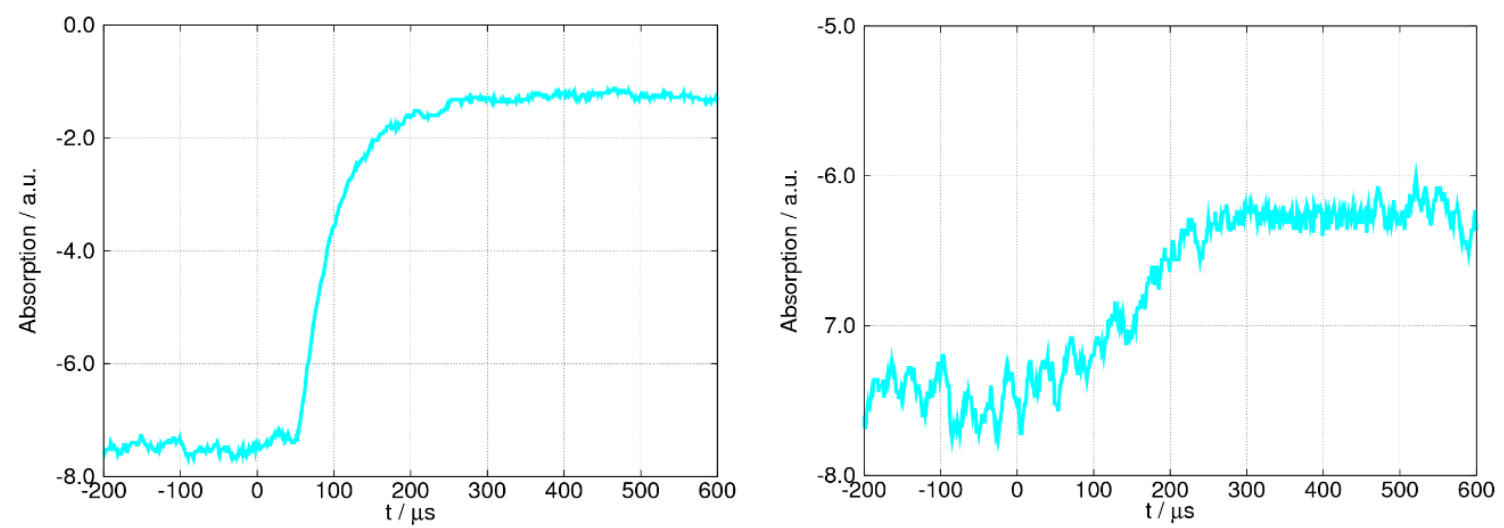

Рис. 1. Типичные профили поглощения атомов О на линии 130.5 нм: а) $T_{5}=2140 \mathrm{~K}, P_{5}=$

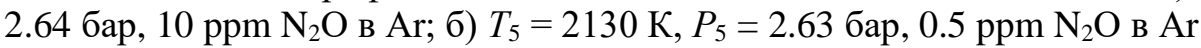




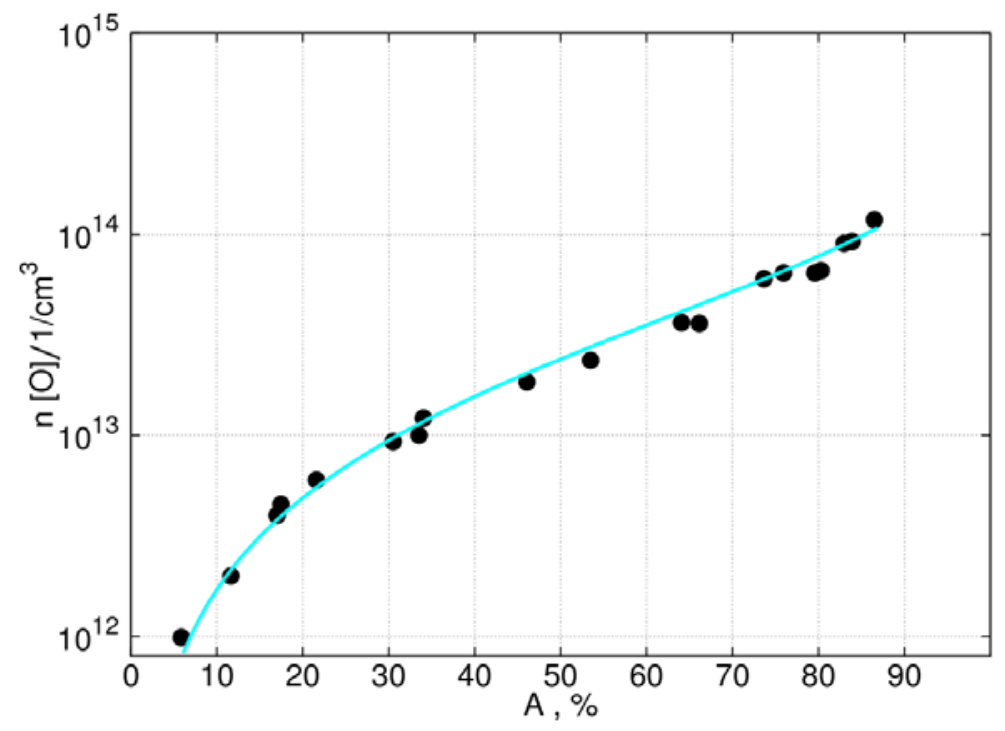

Рис. 2. Калибровочная зависимость для концентрации атома кислорода: $[\mathrm{O}]=f(\mathrm{~A})$, определенная по измерениям в различных смесях $\mathrm{N}_{2} \mathrm{O} / \mathrm{Ar}$ с помощью уравнения (1) при показателе экспоненты $\alpha=0.7$

На рис. 3 показано изменение концентрации атомов кислорода во время диссоциации $\mathrm{N}_{2} \mathrm{O}$, а также результаты моделирования с использованием механизма реакции, приведенные в работе [36], разработанные на основе полного механизма диссоциации $\mathrm{N}_{2} \mathrm{O}$ Коннова и Де Рюйка [39].

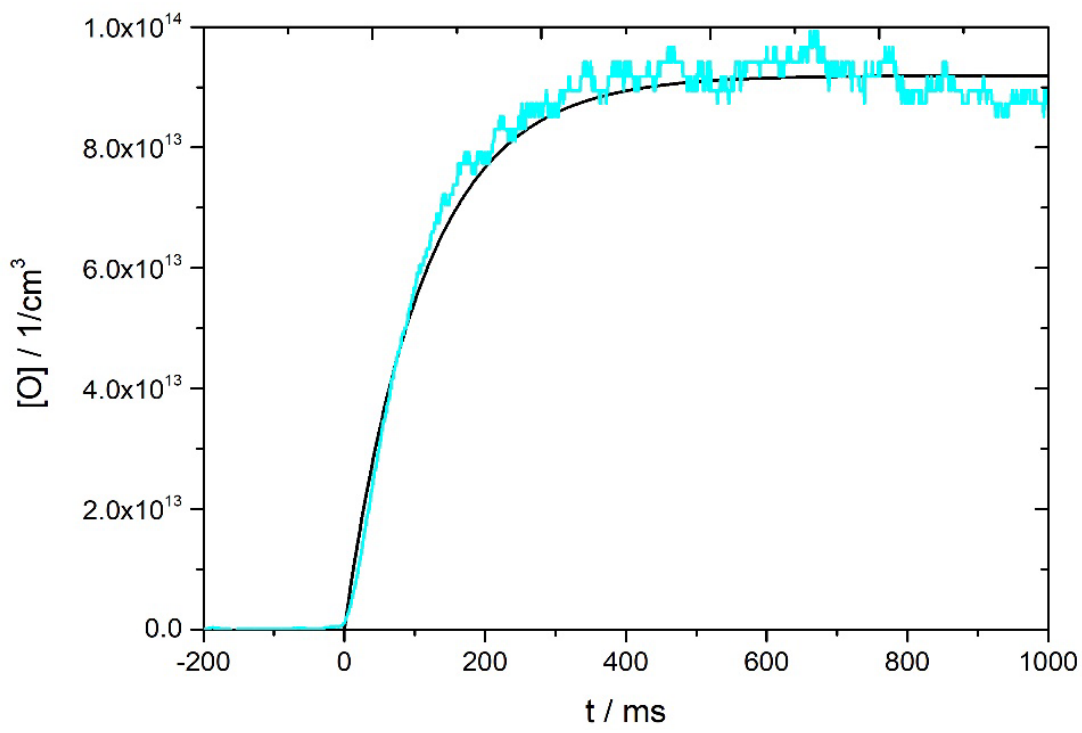

Рис. 3. Типичные экспериментальный (зашумленная кривая) и рассчитанный (сплошная линия) профили концентрации атомов О полученные при 2096 К и 2.63 бар в смеси 10 $\operatorname{ppm~} \mathrm{N}_{2} \mathrm{O}$ в $\mathrm{Ar}$

Для учета добавочного поглощения сигналов в н-бутаноле на линии атома кислорода 130.5 нм была проведена специальная серия экспериментов. Получены сечения поглощения на линии 130.5 нм в смеси 100 ppm н-бутанола в аргоне (рис. 4) при температурах 300-1100 К. Для н-бутанола сечение поглощения на линии 130.5 нм составило $\sigma\left[\mathrm{n}-\mathrm{C}_{4} \mathrm{H}_{9} \mathrm{OH}\right]=3 \cdot 10^{-17}$ $\mathrm{cm}^{2}$ и оказалось постоянным в температурном диапазоне $300-1100 \mathrm{~K}$. При $T_{5}>1100 \mathrm{~K}$ нбутанол, концентрация которого в основной серии экспериментов была ниже на несколько порядков, быстро распадается, т.е. поглощение, как ультрамалых концентраций продуктов распада, так и самого н-бутанола находилось в пределах шума. 


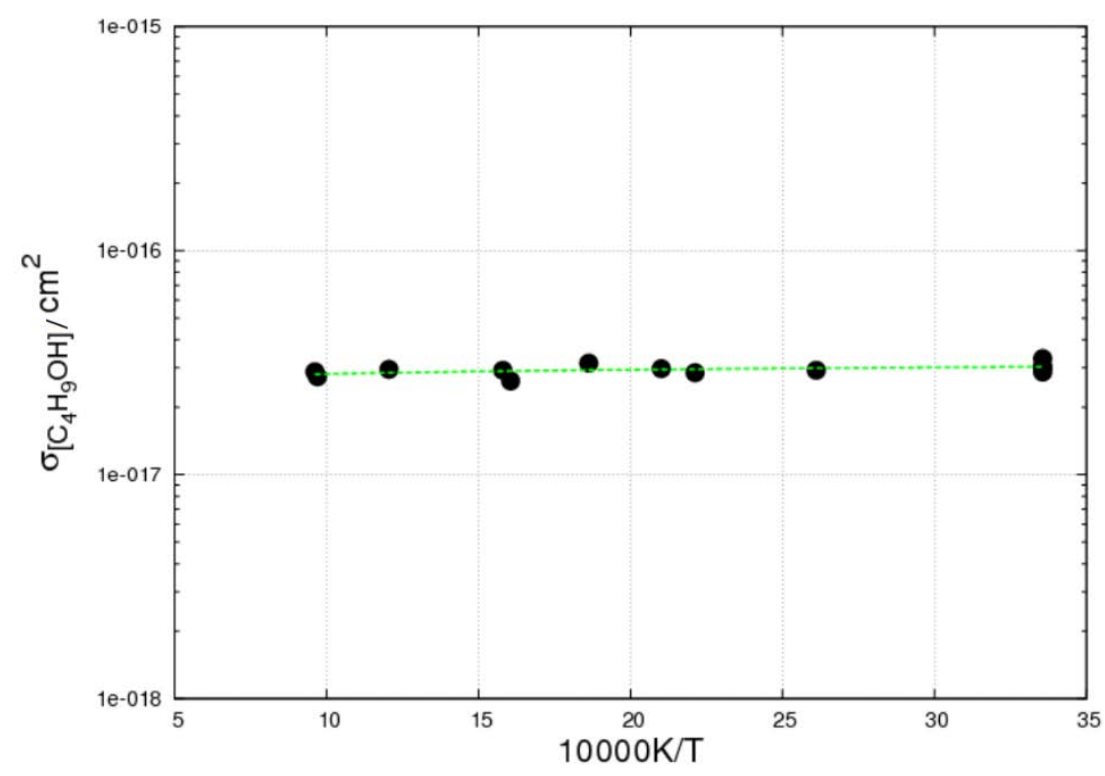

Рис. 4. Сечение поглощения на линии 130.5 нм в смесях н-бутанола с аргоном при температурах 300-1100 K

\section{3. Результаты и обсуждение}

\section{1. Реакция $\mathrm{N}_{2} \mathrm{O}$ с н-бутанолом}

При добавлении н-бутанола к закиси азота профиль концентрации атома кислорода существенно изменялся. На рис. 5 показано сравнение экспериментальных (зашумленные

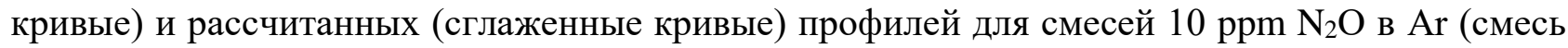
1), 10 ppm $\mathrm{N}_{2} \mathrm{O}+1$ ppm n- $\mathrm{C}_{4} \mathrm{H}_{9} \mathrm{OH}$ в $\mathrm{Ar}$ (смесь 2) и 10 ppm $\mathrm{N}_{2} \mathrm{O}+10$ ppm n- $\mathrm{C}_{4} \mathrm{H}_{9} \mathrm{OH}$ (смесь 3 ). Из всех сигналов вычтено добавочное поглощение, возникающее при начальном наливе смеси в ударную трубу и за падающей ударной волной, с использованием ранее полученного сечения поглощения в н-бутаноле.

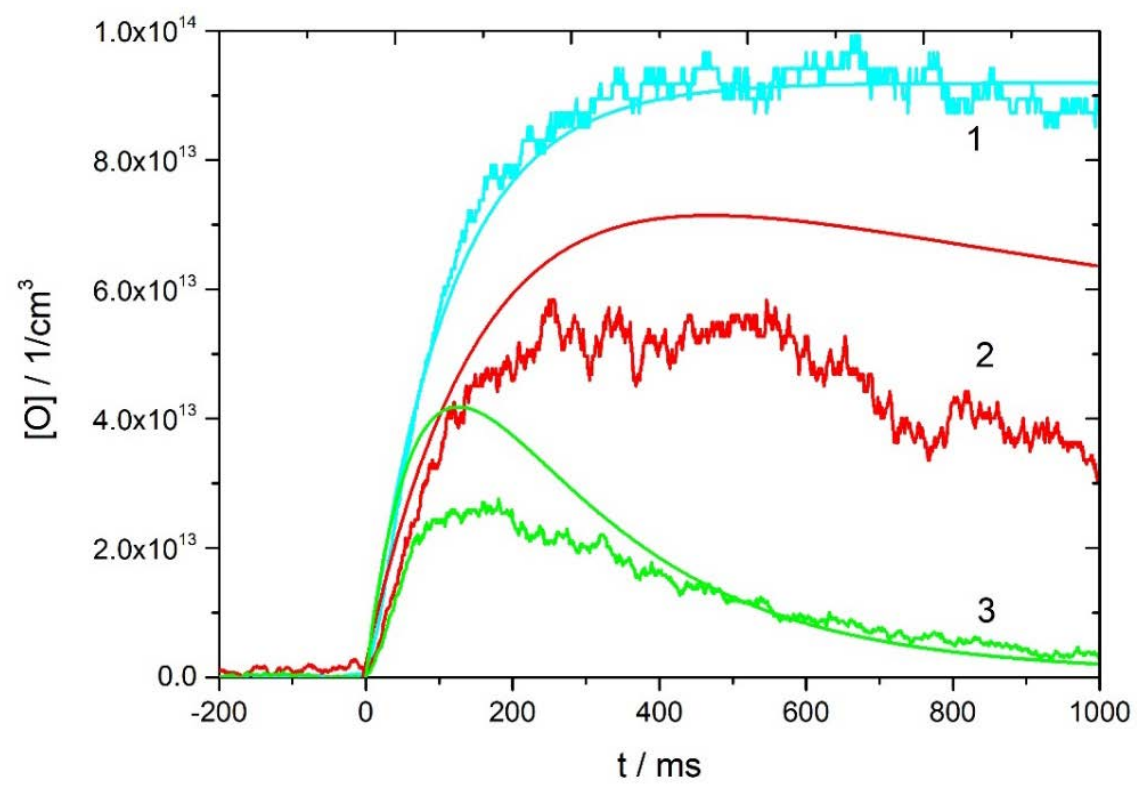

Рис. 5. Типичные экспериментальные (зашумленные кривые) и рассчитанные (сглаженные кривые) профили концентрации атомов О, полученные при $2100 \pm 50 \mathrm{~K}$ и $260 \pm 6$ мбар для смесей: 10 ppm $\mathrm{N}_{2} \mathrm{O}$ в $\mathrm{Ar}(1) ; 10$ ppm $\mathrm{N}_{2} \mathrm{O}+1$ ppm n- $\mathrm{C}_{4} \mathrm{H}_{9} \mathrm{OH}$ в $\mathrm{Ar}$ (2) и 10

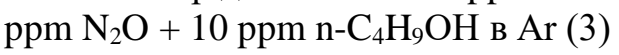


Отметим, что расчеты выхода атомов кислорода, проведенные с использованием различных схем горения н-бутанола [40-41], показывают аналогичные результаты. Поэтому на рис. 5 приведены только расчеты (сглаженные кривые (1-3)), выполненные в соответствии со схемой из работы [40]. Видно значительное расхождение моделирования и экспериментальных результатов для всех смесей, содержащих н-бутанол. Причин таких расхождений может быть несколько: неточности в определении констант скоростей (и/или неоптимизированность этих констант под высокие температуры) отдельных реакций, записанных в схемах работ [40-41]; отсутствие/игнорирование части потенциально-значимых реакций кислорода с производными веществами, являющихся продуктами взаимодействия нбутанола с закисью азота; неточности в механизмах распада как самого н-бутанола, так и его радикалов. Рассмотрим эти причины более подробно.

\section{2. Неопределенность констант скоростей}

На примере типового эксперимента со смесью (2) при параметрах за отраженной ударной волной $T_{5}=2065 \mathrm{~K}, P_{5}=2.439$ бар, описанного кинетической схемой [40], с помощью пакета Chemkin при использовании встроенного алгоритма «Analyze Reaction Path», был проведен анализ временных профилей концентрации атомарного кислорода. Это позволило получить информацию о моменте времени $\tau$, соответствующем появлению или убыли исследуемых атомов/молекул выше (ниже) порога чувствительности, принятом около $10^{4} \mathrm{~cm}^{-3}$ (обычно по шагу расчета, равным $\sim 1$ мкс или более), для каждой реакции, участвующей в кинетическом механизме. Эта информация позволяет выявлять превалирующие и второстепенные пути реакции, время их появления (или вноса существенного влияния) и их точный количественный вклад в тот или иной механизм. Данный анализ выявил более 20 химических реакций с участием атомарного кислорода. Соответственно, оптимизируя константы скорости этих реакций под высокотемпературное окисление, можно добиться улучшения описания экспериментальных кривых моделированием. В табл. 1 приведены основные из них.

Таблица 1

\begin{tabular}{|c|c|}
\hline Химическая реакция & $\tau$, мкс \\
\hline $\mathrm{O}+\mathrm{CH}_{2} \mathrm{O}=\mathrm{OH}+\mathrm{HCO}$ & $<100$ \\
\hline $\mathrm{O}+\mathrm{C}_{2} \mathrm{H}_{4}=\mathrm{CH}_{3}+\mathrm{HCO}$ & $<100$ \\
\hline $\mathrm{O}+\mathrm{C}_{2} \mathrm{H}_{4}=\mathrm{CH}_{2} \mathrm{CHO}+\mathrm{H}$ & $<100$ \\
\hline $\mathrm{OH}+\mathrm{O}=\mathrm{H}+\mathrm{O}_{2}$ & $<100$ \\
\hline $\mathrm{C}_{3} \mathrm{H}_{3}+\mathrm{O}=\mathrm{CH}_{2} \mathrm{O}+\mathrm{C}_{2} \mathrm{H}$ & 120 \\
$\mathrm{O}+\mathrm{C}_{2} \mathrm{H}_{4}=\mathrm{OH}+\mathrm{C}_{2} \mathrm{H}_{3}$ & 150 \\
\hline $\mathrm{O}+\mathrm{CH}_{2} \mathrm{O}=2 \mathrm{H}+\mathrm{CO}_{2}$ & 180 \\
\hline $\mathrm{C}_{2} \mathrm{H}_{2}+\mathrm{O}=\mathrm{HCCO}+\mathrm{H}$ & 200 \\
\hline $\mathrm{CH}_{2}+\mathrm{O}=\mathrm{CO}+\mathrm{H}_{2}$ & 230 \\
\hline $\mathrm{CH}_{2} \mathrm{O}=\mathrm{CO}+\mathrm{H}$ & 250 \\
\hline $\mathrm{O}^{2} \mathrm{H}_{2}=\mathrm{OH}+\mathrm{H}$ & 280 \\
\hline $\mathrm{C}_{2} \mathrm{H}_{2}+\mathrm{O}=\mathrm{CH}_{2}+\mathrm{CO}$ & 310 \\
\hline $\mathrm{O}+\mathrm{CH}_{3}+\mathrm{M}=\mathrm{CH}_{3} \mathrm{O}+\mathrm{M}$ & 500 \\
\hline $\mathrm{O}+\mathrm{CH}_{4}=\mathrm{OH}^{2} \mathrm{CH}$ & 580 \\
\hline $\mathrm{C}_{4} \mathrm{H}_{2}+\mathrm{O}=\mathrm{C}_{3} \mathrm{H}_{2}+\mathrm{CO}$ & 960 \\
\hline
\end{tabular}


Проведена оптимизация констант скоростей основных реакций (табл. 1) под конкретные условия эксперимента. В результате, при расчете в Chemkin, c оптимизированными константами удалось добиться более точного (на 10-20\%) описания экспериментальных данных. Однако, вследствие громоздкости схем (более 12000 реакций в случае схемы [40]), такие изменения могут вызывать немонотонность расчетных кривых. Ситуацию усугубляет большое количество путей реакций, обладающих противоположной чувствительностью [18], конкурирующих за кислород - это значит, что изменения констант скоростей для улучшения описания одних путей реакции ухудшают описание других.

Таким образом, продемонстрировано, что модель окисления н-бутанола может быть изменена в рамках данного подхода лишь незначительно. Для существенного улучшения кинетических схем горения н-бутанола при высоких температурах необходим учет потенциально-значимых реакций кислорода с производными веществами, являющихся продуктами взаимодействия н-бутанола с закисью азота.

\section{3. Дополнительные реакции}

Проведена попытка объяснить расхождение расчетов с экспериментальными данными при учете реакций взаимодействия $\mathrm{N}_{2} \mathrm{O}$ с некоторыми продуктами пиролиза н-бутанола (табл. 2).

Таблица 2

\begin{tabular}{|c|c|c|c|c|}
\hline Источник & $\begin{array}{c}\text { Химическая } \\
\text { реакция }\end{array}$ & Константа скорости & Характер данных & $T, \mathrm{~K} ; P$, бар \\
\hline $\begin{array}{c}\text { Bozzelli, J.W. } \\
1994 \text { [42] }\end{array}$ & $\begin{array}{c}\mathrm{N}_{2} \mathrm{O}+\mathrm{H}=\mathrm{O}+ \\
\mathrm{N}_{2} \mathrm{H}\end{array}$ & $\begin{array}{l}2.18 \times 10^{-8}\left[\mathrm{~cm}^{3} / \text { molecules }\right] \\
\left(T / 298 \mathrm{~K}^{-1.06} \mathrm{e}^{-198}[\mathrm{~kJ} / \mathrm{mole}] / \mathrm{RT}\right.\end{array}$ & $\begin{array}{l}\text { Эксперимент, } \\
\text { РРКМ } \\
\text { экстраполяция }\end{array}$ & $\begin{array}{l}300-4000 \\
1.01\end{array}$ \\
\hline $\begin{array}{c}\text { Bozzelli, J.W. } \\
1994 \text { [42] }\end{array}$ & $\begin{array}{c}\mathrm{N}_{2} \mathrm{O}+\mathrm{H}=\mathrm{NO}+ \\
\mathrm{NH}\end{array}$ & $\begin{array}{l}5.03 \times 10^{-7}\left[\mathrm{~cm}^{3} / \text { molecules] }\right. \\
\left(T / 298 \mathrm{~K}^{-2.16} \mathrm{e}^{-155}[\mathrm{~kJ} / \mathrm{mole}] / \mathrm{RT}\right.\end{array}$ & $\begin{array}{l}\text { Эксперимент, } \\
\text { РРКМ } \\
\text { экстраполяция }\end{array}$ & $\begin{array}{l}300-4000 \\
\quad 1.01\end{array}$ \\
\hline $\begin{array}{c}\text { Bozzelli, J.W. } \\
1994 \text { [42] }\end{array}$ & $\begin{array}{c}\mathrm{N}_{2} \mathrm{O}+\underset{\mathrm{H}}{\mathrm{H}}=\mathrm{OH}+ \\
\mathrm{N}_{2}\end{array}$ & $\begin{array}{c}3.65 \times 10^{-10}\left[\mathrm{~cm}^{3} / \text { molecules }\right] \\
\mathrm{e}^{-70.09}[\mathrm{~kJ} / \mathrm{mole}] / \mathrm{R} T\end{array}$ & $\begin{array}{l}\text { Эксперимент, } \\
\text { РРКМ } \\
\text { экстраполяция }\end{array}$ & $\begin{array}{l}1000-2000 ; \\
1.01\end{array}$ \\
\hline $\begin{array}{l}\text { Starikovskii, } \\
\text { A.Y. } 1994 \text { [43] }\end{array}$ & $\begin{array}{c}\mathrm{CO}+\mathrm{N}_{2} \mathrm{O}=\mathrm{N}_{2}+ \\
\mathrm{CO}_{2}\end{array}$ & $\begin{array}{c}1.66 \times 10^{-12}\left[\mathrm{~cm}^{3} / \text { molecules }\right] \\
\mathrm{e}^{-166}[\mathrm{~kJ} / \mathrm{mole}] / \mathrm{R} T\end{array}$ & $\begin{array}{c}\text { Эксперимент, } \\
\text { прямое измерение }\end{array}$ & $\begin{array}{c}1800-2100 ; \\
-\end{array}$ \\
\hline $\begin{array}{l}\text { Roose, T.R. } \\
1978[44]\end{array}$ & $\begin{array}{c}\mathrm{N}_{2} \mathrm{O}+\mathrm{H}_{2}=\mathrm{H}_{2} \mathrm{O} \\
+\mathrm{N}_{2}\end{array}$ & $\begin{array}{c}5.73 \times 10^{-12}\left[\mathrm{~cm}^{3} / \text { molecules }\right] \\
(T / 298 \mathrm{~K})^{0.50}\end{array}$ & $\begin{array}{c}\text { Теоретический } \\
\text { термохимический } \\
\text { расчет }\end{array}$ & $\begin{array}{l}1700-3000 \\
0.3-0.7\end{array}$ \\
\hline $\begin{array}{c}\text { Trenwith, A.B. } \\
1960 \text { [45] }\end{array}$ & $\begin{array}{l}\mathrm{C}_{2} \mathrm{H}_{4}+\mathrm{N}_{2} \mathrm{O}=\mathrm{N}_{2} \\
\quad+\mathrm{CH}_{3} \mathrm{HCO}\end{array}$ & $\begin{array}{c}1.32 \times 10^{-14}\left[\mathrm{~cm}^{3} / \text { molecules }\right] \\
\mathrm{e}^{-159}[\mathrm{~kJ} / \mathrm{mole}] / \mathrm{RT}\end{array}$ & $\begin{array}{c}\text { Эксперимент, } \\
\text { прямое измерение }\end{array}$ & $\begin{array}{l}828-863 \\
0.13-0.27\end{array}$ \\
\hline $\begin{array}{l}\text { Allen, M.T. } \\
1995 \text { [46] }\end{array}$ & $\begin{array}{c}\mathrm{OH}+\mathrm{N}_{2} \mathrm{O}=\mathrm{N}_{2}+ \\
\mathrm{HO}_{2}\end{array}$ & $9.4 \times 10^{-16}\left[\mathrm{~cm}^{3} /\right.$ molecules $]$ & $\begin{array}{c}\text { Эксперимент, } \\
\text { прямое измерение }\end{array}$ & $\begin{array}{c}1120 ; \\
1.52-10.6\end{array}$ \\
\hline $\begin{array}{c}\text { Zuev, A.P. } 1991 \\
\text { [47] }\end{array}$ & $\begin{array}{c}\mathrm{N}_{2} \mathrm{O}+\mathrm{O}=\mathrm{NO}+ \\
\mathrm{NO}\end{array}$ & $\begin{array}{c}1.49 \times 10^{-10}\left[\mathrm{~cm}^{3} / \text { molecules }\right] \\
\mathrm{e}^{-117[\mathrm{~kJ} / \mathrm{mole}] / \mathrm{RT}}\end{array}$ & $\begin{array}{c}\text { Эксперимент, } \\
\text { прямое измерение }\end{array}$ & $\begin{array}{c}1750-3300 ; \\
2.43-23.3\end{array}$ \\
\hline $\begin{array}{c}\text { Zuev, A.P. } 1991 \\
\text { [47] }\end{array}$ & $\begin{array}{c}\mathrm{N}_{2} \mathrm{O}+\mathrm{O}=\mathrm{N}_{2}+ \\
\mathrm{O}_{2}\end{array}$ & $\begin{array}{c}\text { 8.32x10 } 0^{-12}\left[\mathrm{~cm}^{3} / \mathrm{molecules}\right] \\
\mathrm{e}^{-116[\mathrm{~kJ} / \mathrm{mole}] / \mathrm{RT}}\end{array}$ & $\begin{array}{c}\text { Эксперимент, } \\
\text { прямое измерение }\end{array}$ & $\begin{array}{c}1750-3300 ; \\
2.53-23.3\end{array}$ \\
\hline $\begin{array}{c}\text { Kenwright, R. } \\
1959 \text { [48] }\end{array}$ & $\begin{array}{c}\mathrm{N}_{2} \mathrm{O}+\mathrm{C}_{2} \mathrm{H}_{5}= \\
\mathrm{CH}_{3} \mathrm{CH}_{2} \mathrm{O} \\
+\mathrm{N}_{2} \\
\end{array}$ & $\begin{array}{c}3.32 \times 10^{-24}\left[\mathrm{~cm}^{3} / \mathrm{molecule}\right] \\
\mathrm{e}^{35.59[\mathrm{~kJ} / \mathrm{mole}] / \mathrm{R} T}\end{array}$ & $\begin{array}{c}\text { Эксперимент, } \\
\text { прямое измерение }\end{array}$ & $\begin{array}{l}826-861 \\
0.13-0.27\end{array}$ \\
\hline
\end{tabular}


Заметных изменений в форме расчетных кривых при добавлении к расчетной схеме дополнительных реакций (табл. 2) обнаружено не было. Поэтому, чтобы улучшить согласие между моделированием и экспериментальными данными, в кинетические схемы были также добавлены реакции взаимодействия продуктов пиролиза $\mathrm{N}_{2} \mathrm{O}$ и n- ${ }_{4} \mathrm{H}_{9} \mathrm{OH}$ (табл. 3).

Таблица 3

\begin{tabular}{|c|c|c|c|c|}
\hline Источник & $\begin{array}{l}\text { Химическая } \\
\text { реакция }\end{array}$ & Константа скорости & Характер данных & $T, \mathrm{~K} ; P$, бар \\
\hline $\begin{array}{l}\text { Tomeczek, J. } \\
2003 \text { [49] }\end{array}$ & $\begin{array}{c}\mathrm{CH}_{3} \mathrm{~N}+\mathrm{O}=\mathrm{CH}_{2} \mathrm{O} \\
+\mathrm{NH}\end{array}$ & $\begin{array}{l}\text { 1.62x10 } 10^{4}\left[\mathrm{~cm}^{3} / \text { molecules] }\right. \\
(T / 298 \mathrm{~K})^{1.25} \mathrm{e}^{-20.00[\mathrm{~kJ} / \mathrm{mole}] / \mathrm{R} T}\end{array}$ & $\begin{array}{c}\text { Теоретический } \\
\text { расчет }\end{array}$ & - \\
\hline $\begin{array}{l}\text { Tomeczek, } \\
\text { J.2003 [49] }\end{array}$ & $\begin{array}{c}\mathrm{CH}_{3} \mathrm{~N}+\mathrm{H}=\mathrm{CH}_{2} \mathrm{~N} \\
+\mathrm{H}_{2}\end{array}$ & $\begin{array}{c}\text { 8.32x10 } 0^{3}\left[\mathrm{~cm}^{3} / \text { molecules }\right] \\
\mathrm{e}^{-10.00[\mathrm{~kJ} / \mathrm{mole}] / \mathrm{R} T}\end{array}$ & $\begin{array}{l}\text { Теоретический } \\
\text { расчет }\end{array}$ & - \\
\hline $\begin{array}{l}\text { Tomeczek, } \\
\text { J.2003 [49] }\end{array}$ & $\begin{array}{c}\mathrm{CH}_{2} \mathrm{~N}+\mathrm{O}=\mathrm{HCN} \\
+\mathrm{OH}\end{array}$ & $5.0 \times 10^{7}\left[\mathrm{~cm}^{3} /\right.$ molecules $]$ & $\begin{array}{l}\text { Теоретический } \\
\text { расчет }\end{array}$ & - \\
\hline $\begin{array}{l}\text { Tomeczek, J. } \\
2003 \text { [49] }\end{array}$ & $\begin{aligned} \mathrm{CH}_{3} \mathrm{~N} & +\mathrm{O}=\mathrm{CH}_{2} \mathrm{~N} \\
& +\mathrm{OH}\end{aligned}$ & $\begin{array}{c}1.62 \times 10^{4}\left[\mathrm{~cm}^{3} / \text { molecules }\right] \\
(T / 298 \mathrm{~K})^{1.25} \mathrm{e}^{-20.00[\mathrm{~kJ} / \mathrm{mole}] / \mathrm{RT} T}\end{array}$ & $\begin{array}{l}\text { Теоретический } \\
\text { расчет }\end{array}$ & - \\
\hline $\begin{array}{l}\text { Tomeczek, J. } \\
2003 \text { [49] }\end{array}$ & $\begin{array}{c}\mathrm{CH}_{3}+\mathrm{N}_{2} \mathrm{O}=\mathrm{CH}_{3} \mathrm{~N} \\
+\mathrm{NO}\end{array}$ & $\begin{array}{l}6.73 \times 10^{4}\left[\mathrm{~cm}^{3} / \text { molecules] }\right. \\
(T / 298 \mathrm{~K})^{2.00} \mathrm{e}^{-127}[\mathrm{~kJ} / \mathrm{mole}] / \mathrm{RT}\end{array}$ & $\begin{array}{l}\text { Теоретический } \\
\text { расчет }\end{array}$ & - \\
\hline $\begin{array}{l}\text { Tomeczek, J. } \\
2003 \text { [49] }\end{array}$ & $\begin{array}{c}\mathrm{CH}_{3}+\mathrm{N}_{2} \mathrm{O}=\mathrm{CH}_{3} \mathrm{O} \\
+\mathrm{N}_{2}\end{array}$ & $\begin{array}{c}1.66 \times 10^{-9}\left[\mathrm{~cm}^{3} / \text { molecule } \mathrm{s}\right] \\
\mathrm{e}^{-119}[\mathrm{~kJ} / \mathrm{mole}] / \mathrm{RT}\end{array}$ & Эксперимент & $\begin{array}{l}1000-2000 \\
1.01\end{array}$ \\
\hline $\begin{array}{l}\text { Bauerle, S. } \\
1995 \text { [50] }\end{array}$ & $\begin{array}{c}\mathrm{NO}+\mathrm{CH}_{2}=\mathrm{HCN} \\
+\mathrm{OH}\end{array}$ & $\begin{array}{c}8.32 \times 10^{-13}\left[\mathrm{~cm}^{3} / \text { molecule s }\right] \\
\mathrm{e}^{-11.97[ \pm 2.04 \mathrm{~kJ} / \mathrm{mole}] / \mathrm{R} T}\end{array}$ & $\begin{array}{c}\text { Эксперимент, } \\
\text { прямое измерение }\end{array}$ & $\begin{array}{l}1100-2600 \\
1.01\end{array}$ \\
\hline $\begin{array}{l}\text { Dagaut, P. } \\
1999[51]\end{array}$ & $\begin{array}{l}\mathrm{NO}+\mathrm{HCCO}= \\
\mathrm{HCN}+\mathrm{CO}_{2}\end{array}$ & $\begin{array}{c}4.65 \times 10^{-11}\left[\mathrm{~cm}^{3} / \text { molecules }\right] \\
\mathrm{e}^{-7.00[\mathrm{~kJ} / \mathrm{mole}] / \mathrm{R} T}\end{array}$ & $\begin{array}{l}\text { Эксперимент, } \\
\text { хроматография }\end{array}$ & $\begin{array}{c}1050-1300 ; \\
1.01\end{array}$ \\
\hline $\begin{array}{l}\text { Chakraborty, } \\
\text { D. } 1999 \text { [52] }\end{array}$ & $\begin{array}{l}\mathrm{N}_{2} \mathrm{O}+\mathrm{CH}_{2} \mathrm{~N}= \\
\mathrm{CH}_{2} \mathrm{~N}_{2}+\mathrm{NO}\end{array}$ & $\begin{array}{c}9.93 \times 10^{-12}\left[\mathrm{~cm}^{3} / \text { molecules }\right] \\
\mathrm{e}^{-183[\mathrm{~kJ} / \mathrm{mole}] / \mathrm{RT}}\end{array}$ & $\begin{array}{l}\text { Эксперимент, } \\
\text { РРКМ } \\
\text { экстраполяция }\end{array}$ & $\begin{array}{c}1000-3000 ; \\
-\end{array}$ \\
\hline $\begin{array}{l}\text { Cohen, N. } \\
1991 \text { [53] }\end{array}$ & $\mathrm{NH}+\mathrm{O}=\mathrm{NO}+\mathrm{H}$ & $1.16 \times 10^{-10}\left[\mathrm{~cm}^{3} /\right.$ molecules $]$ & $\begin{array}{c}\text { Обзорная } \\
\text { литература }\end{array}$ & $\begin{array}{c}250-3000 \\
-\end{array}$ \\
\hline $\begin{array}{c}\text { Hack, W. } 1994 \\
{[54]}\end{array}$ & $\mathrm{NH}+\mathrm{O}=\mathrm{OH}+\mathrm{N}$ & $1.66 \times 10^{-13}\left[\mathrm{~cm}^{3} /\right.$ molecules $]$ & $\begin{array}{c}\text { Эксперимент, } \\
\text { лазерная } \\
\text { флуоресценция }\end{array}$ & $\begin{array}{c}298 \\
0.015\end{array}$ \\
\hline $\begin{array}{l}\text { McCullough, } \\
\text { R.W. 1977 } \\
\text { [55] }\end{array}$ & $\mathrm{NO}+\mathrm{O}=\mathrm{O}_{2}+\mathrm{N}$ & $\begin{array}{l}\text { 8.52x10-13 }\left[\mathrm{cm}^{3} / \text { molecules }\right] \\
(T / 298 \mathrm{~K})^{1.00} \mathrm{e}^{-161[\mathrm{~kJ} / \mathrm{mole} / \mathrm{R} T}\end{array}$ & $\begin{array}{l}\text { Эксперимент, } \\
\text { хроматография }\end{array}$ & $\begin{array}{l}1750-2100 ; \\
1.01\end{array}$ \\
\hline $\begin{array}{c}\text { Murrell, J.N. } \\
1986 \text { [56] }\end{array}$ & $\mathrm{CH}+\mathrm{O}=\mathrm{OH}+\mathrm{C}$ & $\begin{array}{c}2.52 \times 10^{-11}\left[\mathrm{~cm}^{3} / \text { molecules }\right] \\
\mathrm{e}^{-19.79[\mathrm{~kJ} / \mathrm{mole}] / \mathrm{RT}}\end{array}$ & $\begin{array}{c}\text { Теоретический } \\
\text { расчет }\end{array}$ & $\begin{array}{l}10.00- \\
6000 ;-\end{array}$ \\
\hline $\begin{array}{l}\text { Tsuboi, T. } \\
1981 \text { [57] }\end{array}$ & $\begin{array}{c}\mathrm{O}+\mathrm{CH}_{2}=\mathrm{HCO}+ \\
\mathrm{H}\end{array}$ & $5.01 \times 10^{-11}\left[\mathrm{~cm}^{3} /\right.$ molecules $]$ & $\begin{array}{c}\text { Теоретический } \\
\text { термохимический } \\
\text { расчет }\end{array}$ & $\begin{array}{l}1200-1800 \\
0.2-5\end{array}$ \\
\hline $\begin{array}{l}\text { Peeters, J. } \\
1995 \text { [58] }\end{array}$ & $\begin{array}{c}\mathrm{O}+\mathrm{HCCO}=\mathrm{CO}_{2} \\
+\mathrm{CH}\end{array}$ & $\begin{array}{c}4.9 \times 10^{-11}\left[ \pm 2.66 \times 10^{-11}\right. \\
\mathrm{cm}^{3} / \text { molecules] } \mathrm{e}^{-4.66[ \pm 2.51} \\
\mathrm{kJ} / \mathrm{mole}] / \mathrm{RT}\end{array}$ & $\begin{array}{c}\text { Эксперимент, } \\
\text { масс- } \\
\text { спектрометрия }\end{array}$ & $\begin{array}{l}450-960 \\
0.0027\end{array}$ \\
\hline
\end{tabular}


Результаты расчетов, проведенных с учетом этих дополнительных реакций (табл. 3) к ранее использованным кинетическим схемам представлены на рис. 6-9. Отметим, что экспериментальный профиль имеет меньшую скорость подъема концентрации кислорода, чем расчетный профиль в начальные моменты времени на рис. 6-9, что может быть отнесено к недостаточному пространственно-временному разрешению проведенных измерений (10-15 мкс).
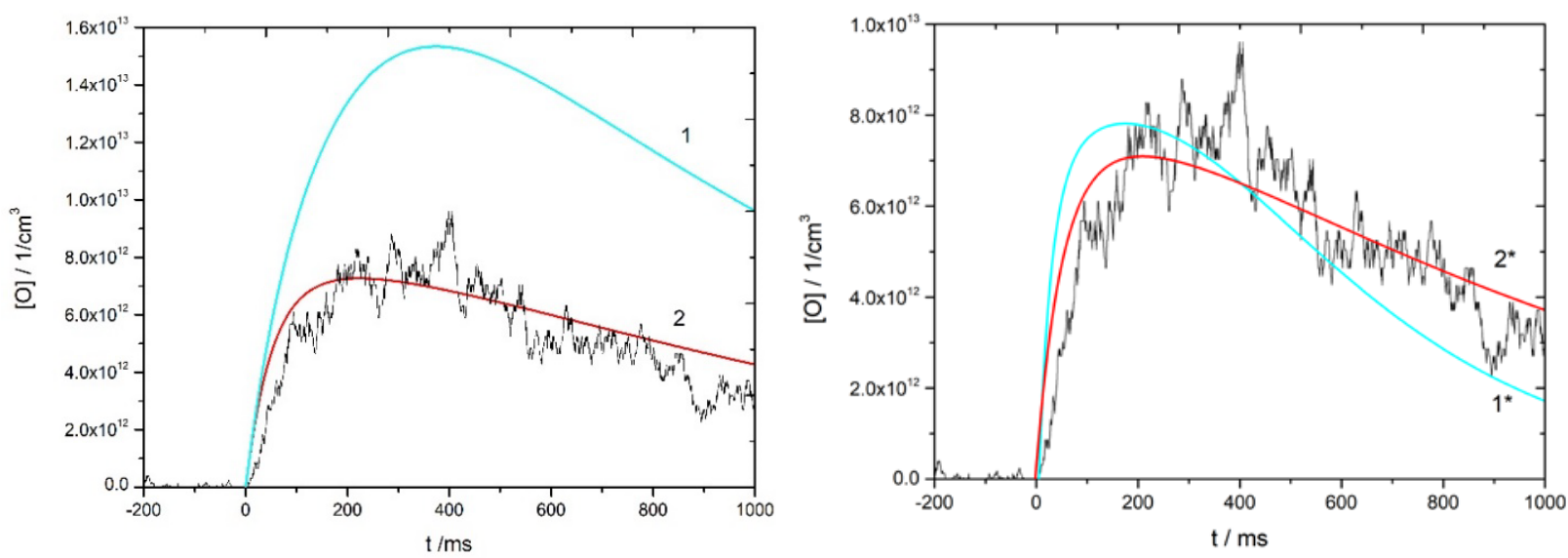

Рис. 6. Расчетные профили концентрации атомов О при $T_{5}=1825 \mathrm{~K}, P_{5}=2.540$ бар в смеси 10 ppm $\mathrm{N}_{2} \mathrm{O}+10 \mathrm{ppm}$ n- $\mathrm{C}_{4} \mathrm{H}_{9} \mathrm{OH}:$ а) кинетические модели в оригинальном виде, голубая линия $1-$ Ranzi et al. [40], красная линия 2 - Sarathy et al. [41]; б) кинетические модели с учетом добвленных реакций (3), голубая линия $1^{*}$ - Ranzi et al. [40], красная линия $2^{*}$ - Sarathy et al. [41]
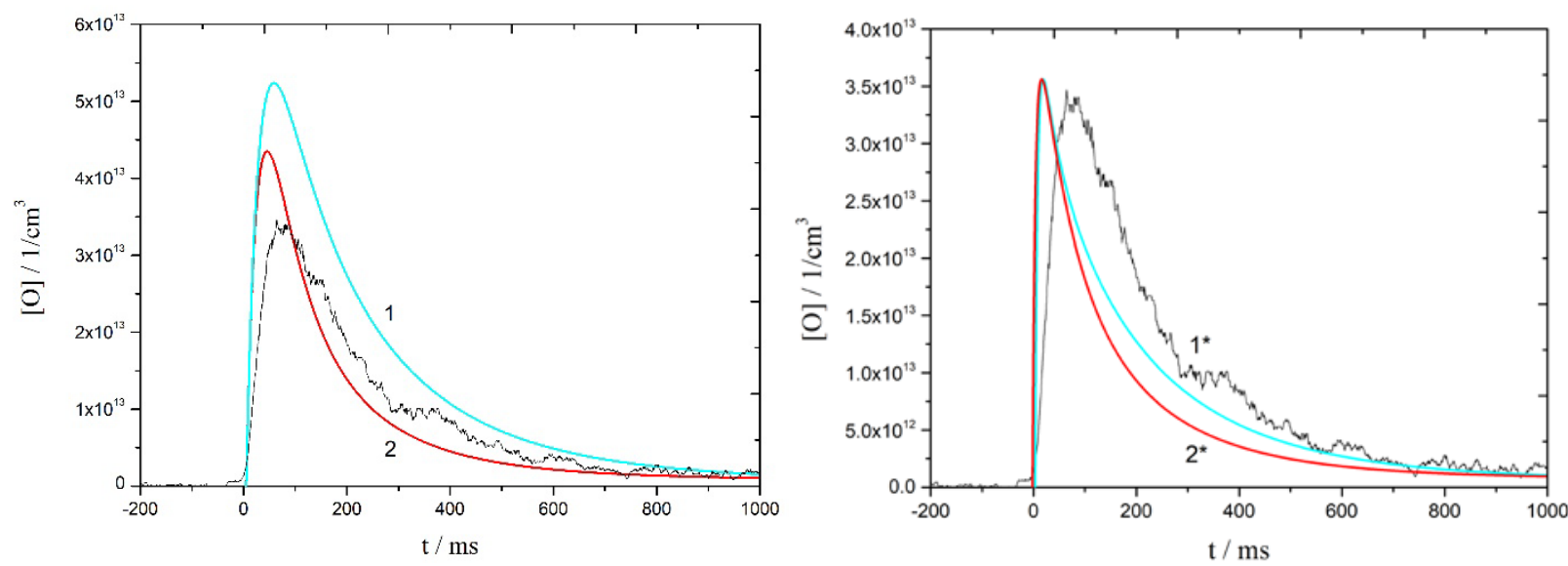

Рис. 7. Расчетные профили концентрации атомов $\mathrm{O}$ при $T_{5}=2393 \mathrm{~K}, P_{5}=2.380$ бар в смеси 10

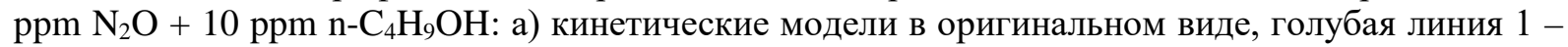
Ranzi et al. [40], красная линия 2 - Sarathy et al. [41]; б) кинетические модели с учетом добавленных реакций (табл. 3), голубая линия $1^{*}$ - Ranzi et al. [40], красная линия $2^{*}$ - Sarathy et al. [41] 

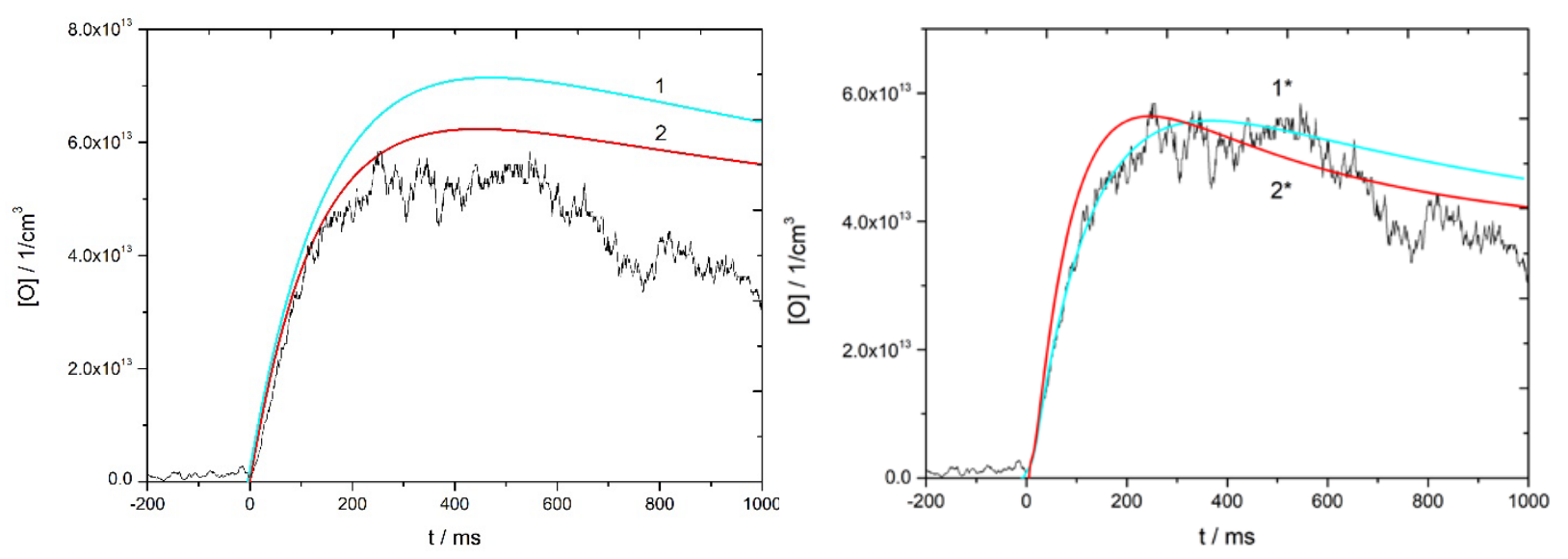

Рис. 8. Расчетные профили концентрации атомов О при $T_{5}=2036 \mathrm{~K}, P_{5}=2.548$ бар в смеси 10

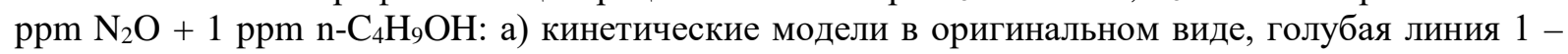
Ranzi et al. [40], красная линия 2 - Sarathy et al. [41]; б) кинетические модели с учетом добавленных реакций (табл. 3), голубая линия $1^{*}$ - Ranzi et al. [40], красная линия $2^{*}$ - Sarathy et al. [41]
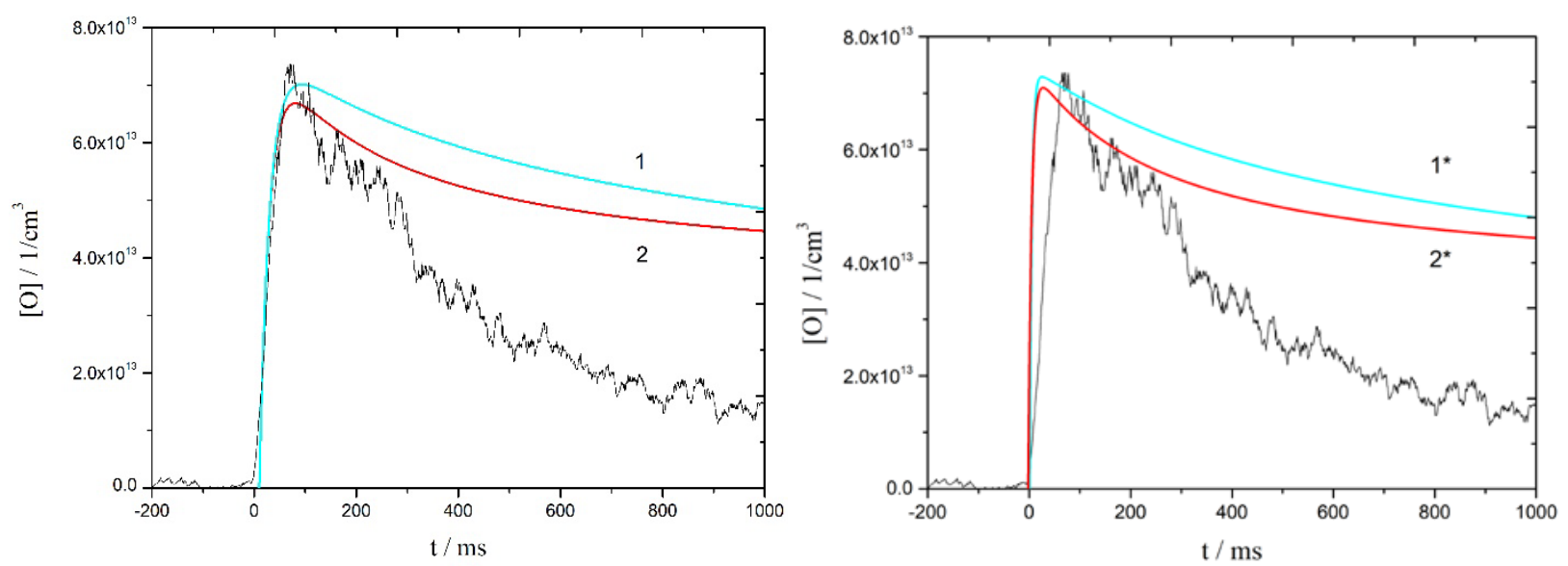

Рис. 9. Расчетные профили концентрации атомов О при $T_{5}=2432 \mathrm{~K}, P_{5}=2.466$ бар в смеси 10 ppm $\mathrm{N}_{2} \mathrm{O}+1$ ppm n- $\mathrm{C}_{4} \mathrm{H}_{9} \mathrm{OH}$ : а) кинетические модели в оригинальном виде, голубая линия 1 Ranzi et al. [40], красная линия 2 - Sarathy et al. [41]; б) кинетические модели с учетом добавленных реакций (табл. 3), голубая линия $1^{*}$ - Ranzi et al. [40], красная линия $2^{*}$ - Sarathy et al. [41]

При этом хорошо видно, что с добавлением реакций взаимодействия продуктов пиролиза $\mathrm{N}_{2} \mathrm{O}$ и n- $\mathrm{C}_{4} \mathrm{H}_{9} \mathrm{OH}$ (табл. 3), удалось добиться значительного улучшения в описании экспериментальных данных во всем исследуемом диапазоне температур для смеси 10 ppm $\mathrm{N}_{2} \mathrm{O}+10$ ppm н-бутанола (рис. 6, 7), однако для смеси 10 ppm $\mathrm{N}_{2} \mathrm{O}+1$ ppm н-бутанола, удалось описать экспериментальные данные только при температурах $\leq 2000$ К (рис. 8); при температурах выше 2000 К улучшить модель удалось лишь незначительно (рис. 9).

\section{4. Мономолекулярная диссоциация н-бутанола}

В работе [41] уже указывалось, что в высокотемпературном режиме преобладают мономолекулярные реакции. Авторами было предложено усовершенствование схемы [31] путем изменения скорости мономолекулярного разложения н-бутанола, предложенного Black et al. [3], где она в значительной степени зависела от давления. Следовательно, обновленная модель использует экспериментально определенные константы скорости n$\mathrm{C}_{4} \mathrm{H}_{9} \mathrm{OH}+(\mathrm{M})$, из работы Tsang et al. [12], первого порядка, что, по-видимому позволило получить лучшее согласие с экспериментальными данными, приведенным на рис. 6-9. 
При рассмотрении рис. 9, нетрудно заметить, что согласие между расчетными и экспериментальными данными наблюдается только в первые 100 микросекунд, отражающие диссоциацию $\mathrm{N}_{2} \mathrm{O}$, далее наблюдается потребление кислорода в количествах, существенно отличающихся от расчетных кинетических схем [40, 41]. Интересен и тот факт, что, при столь низкой концентрации н-бутанола, добавление реакций как из (табл. 2), так и из (табл. 3), и уточнение констант скоростей ключевых реакций с атомарным кислородом практически не повлияли на характер кривых 1, 2 и 1*, 2*. Это согласуется с данными из работы [41] и может говорить о доминировании при столь малых концентрациях реакций первичного мономолекулярного распада биотоплива, когда бимолекулярные реакции при таком разбавлении смеси протекают крайне медленно. Причем, с увеличением температуры данное расхождение лишь увеличивается. Потеря гибкости кинетических схем для смесей с ультрамалыми концентрациями n- $\mathrm{C}_{4} \mathrm{H}_{9} \mathrm{OH}$ в высокотемпературном диапазоне (более $2000 \mathrm{~K}$ ) указывает на незавершенность существующих кинетических механизмов не только при учете взаимодействия продуктов исходных веществ, но и при их первичной мономолекулярной диссоциации.

\section{4. Заключение}

В работе проведены первые прямые измерения временных профилей концентрации атомов $\mathrm{O}$ в процессе окисления н-бутанола за отраженными ударными волнами в широком диапазоне температур и давлений с использованием АРАС-метода. В результате экспериментального и численного исследований получены новые данные о кинетике реакций н-бутанола с кислородом и предложен ряд оптимизирующих дополнений к существующим кинетическим схемам. В ходе сравнения полученных экспериментальных данных с актуальными кинетическими схемами обнаружены принципиальные несоответствия в смесях с ультрамалым содержанием $\mathrm{n}-\mathrm{C}_{4} \mathrm{H}_{9} \mathrm{OH}$, в связи с чем выдвинуты предположения о роли моно и бимолекулярных реакций в процессе окисления сложных спиртов.

\section{Благодарности и ссылки на гранты}

Исследование поддержано грантами РФФИ 17-08-01303 и 18-38-20085.

\section{Литература}

1. Kohse-Höinghaus K., Oßwald P., Cool T. A., Kasper T., Hansen N., Qi F., Westbrook C. K., Westmoreland P. R. Biofuel combustion chemistry: From ethanol to biodiesel // Angew. Chem., Int. Ed. - 2010. - Vol. 49 (21). - P. 3572-3597.

2. Moss J. T., Berkowitz A. M., Oehlschlaeger M. A., Biet J., Warth V., Glaude P. A., Battin-Leclerc F. An experimental and kinetic modeling study of the oxidation of the four isomers of butanol // J. Phys. Chem. A. - 2008. - Vol. 112 (43). - P. 10843-10855.

3. Black G., Curran H. J., Pichon S., Simmie J. M., Zhukov V. Bio-butanol: Combustion properties and detailed chemical kinetic model // Combust. Flame. - 2010. - Vol. 157 (2). - P. 363-373.

4. Durre P. Biobutanol: an attractive biofuel // Biotechnol J. - 2007. - Vol. 2. - P. 1525-1534, doi:10.1002/200700169.

5. Sarathy S., Oßwald P., Hansen N., Kohse-Höinghaus K. Alcohol combustion chemistry // Progress in Energy and Combustion Science. - 2014. - Vol. 44. - P. 40-102.

6. Black G., Curran H. J., Pichon S., Simmie J. M., Zhukov V. Bio-butanol: Combustion properties and detailed chemical kinetic model // Comb. Flame. -2010. - Vol. 157. - P. 363-373.

7. Jerzy M., John M., Henry J. Curran The elimination of water from a conformationally complex alcohol: A computational study of the gas phase dehydration of n-butanol // Journal of Molecular Structure. - 2009. - Vol. 928. - P. 149-157. 
8. Michael R., Kevin M., Steven P., Guy B., William H. Green Comprehensive reaction mechanism for n-butanol pyrolysis and combustion // Comb. Flame. - 2011. - Vol. 158. - P. 16-41.

9. Nigam P.S., Singh A. Production of liquid biofuels from renewable resources // Prog. Energy Combust. Sci. - 2011. - Vol. 30. - P. 37-52.

10. Cai J., Zhang L., Zhang F., Wang Z., Cheng Z., Yuan W., Qi F. Experimental and Kinetic Modeling Study of n-Butanol Pyrolysis and Combustion // Energy and Fuels. - 2010. - Vol. 102. - P. 223-239.

11. Frassoldati A., Grana R., Faravelli T., Ranzi E., Oßwald P., Kohse-Höinghaus K. Detailed kinetic modeling of the combustion of the four butanol isomers in premixed low-pressure flames // Comb. Flame. - 2012. Vol. 159. - P. 2295-2311.

12. Claudette M., Tsang W. Shock Tube Study on the Thermal Decomposition of n-Butanol // The Journal of Physical Chemistry. - 2003. - Vol. 109. - P. 434-451.

13. Charles K. Westbrook Biofuels Combustion // Annu. Rev. Phys. Chem. - 2013. - Vol. 64. - P. 201-19, doi.org/10.1146/annurev-physchem-040412-110009.

14. Davidson D. F., Ranganath K. Y., Lam M., Liaw M., Hong Z., Hanson R. K. Ignition delay time measurements of normal alkanes and simple oxygenates // Journal of Propulsion and Power. - 2010. Vol. 26 (2). - P. 280-287, doi:10.2514/1.44034.

15. Heufer K. A., Fernandes R. X., Olivier H., Beeckmann, J., Rohl O., Peters N. Shock tube investigations of ignition delays of n-butanol at elevated pressures between 770 and $1250 \mathrm{~K} / /$ Proc. Combust. Inst. - 2011. - Vol. 33 (1). - P. 359-366.

16. Vranckx S., Heufer K. A., Lee C., Olivier H., Schill L., Kopp W. A., Leonhard K., Taatjes C. A., Fernandes R. X. Role of peroxy chemistry in the high-pressure ignition of n-butanol Experiments and detailed kinetic modelling // Combust. Flame. - 2011. - Vol. 158 (8). - P. 1444-1455.

17. Weber B. W., Kumar K., Zhang Y., Sung C. J. Autoignition of n-butanol at elevated pressure and lowto-intermediate temperature // Combust. Flame. - 2011. - Vol. 158 (5). - P. 809-819.

18. Stranic I., Chase D. P., Harmon J. T., Yang S., Davidson D. F., Hanson R. K. Shock tube measurements of ignition delay times for the butanol isomers // Combust. Flame. - 2012. - Vol. 159 (2). - P. 516-527.

19. Noorani K. E., Akih-Kumgeh B., Bergthorson J. M. Comparative high temperature shock tube ignition of $\mathrm{C}_{1}-\mathrm{C}_{4}$ primary alcohols // Energy Fuels. - 2010. - Vol. 24 (11). - P. 5834-5843.

20. Karwat D. M., Wagnon S. W., Teini P. D., Wooldridge M. S. On the chemical kinetics of n-butanol: Ignition and speciation studies // J. Phys. Chem. A. - 2011. - Vol. 115 (19). - P. 4909-4921.

21. Gu X. L., Huang Z. H., Li Q. Q., Tang C. L. Measurements of laminar burning velocities and Markstein lengths of n-butanol-air premixed mixtures at elevated temperatures and pressures // Energy Fuels. - 2009. - Vol. 23 (10). - P. 4900-4907.

22. Veloo P. S., Egolfopoulos F. N. Flame propagation of butanol isomers/air mixtures // Proc. Combust. Inst. - 2011. - Vol. 33 (1). - P. 987-993.

23. Gu X. L., Li Q. Q., Huang Z. H. Laminar burning characteristics of diluted n-butanol/air mixtures. Combust. Sci. Technol. - 2011. - Vol. 183 (12). - P. 1360-1375.

24. Gu X. L., Huang Z. H., Wu S., Li Q. Q. Laminar burning velocities and flame instabilities of butanol isomers-air mixtures // Combust. Flame. - 2010. - Vol. 157 (12). - P. 2318-2325.

25. Harper M. R., Van Geem K. M., Pyl S. P., Marin G. B., Green W. H. Comprehensive reaction mechanism for n-butanol pyrolysis and combustion // Combust. Flame. - 2011. - Vol. 158 (1). - P. 1641.

26. Dagaut P., Sarathy S. M., Thomson M. J. A chemical kinetic study of n-butanol oxidation at elevated pressure in a jet stirred reactor // Proc. Combust. Inst. - 2009. - Vol. 32 (1). - P. 229-237.

27. Sarathy S. M., Thomson M. J., Togbe C., Dagaut P., Halter F., Mounaim-Rousselle C. An experimental and kinetic modeling study of n-butanol combustion // Combust. Flame. - 2009. - Vol. 156 (4). - P. 852-864. 
28. Yang B., Oßwald P., Li Y. Y., Wang J., Wei L. X., Tian Z. Y., Qi F., Kohse-Höinghaus K. Identification of combustion intermediates in isomeric fuel-rich premixed butanol-oxygen flames at low pressure // Combust. Flame. - 2007. - Vol. 148 (4). - P. 198-209.

29. Hansen N., Harper M. R., Green W. H. High-temperature oxidation chemistry of n-butanol Experiments in low-pressure premixed flames and detailed kinetic modeling // Phys. Chem. Chem. Phys. - 2011. - Vol. 13 (45). - P. 20262-20274.

30. Oßwald P., Guldenberg H., Kohse-Höinghaus K., Yang B., Yuan T., Qi F. Combustion of butanol isomers A detailed molecular beam mass spectrometry investigation of their flame chemistry // Combust. Flame. - 2011. - Vol. 158 (1). - P. 2-15.

31. Sarathy S. M., Vranckx S., Yasunaga K., Mehl M., Oßwald P., Metcalfe W. K., Westbrook C. K., Pitz W. J., Kohse-Höinghaus K., Fernandes R. X., Curran H. J. A comprehensive chemical kinetic combustion model for the four butanol isomers // Combust. Flame. - 2012. - Vol. 159 (6). - P. 20282055.

32. Drakon A. V., Emelianov A. V., Eremin A. V., Yatsenko P. I. Study of trifluoromethane dissociation within wide pressure and temperature ranges by molecular resonance absorption spectroscopy // High Temp. - 2017. - Vol. 55. - P. 239-245.

33. Emelianov A. V., Eremin A. V., Yatsenko P. I. Experimental study of chlorine atom interaction with acetylene behind shock waves // High Temp. - 2017. - Vol. 55. - P. 788-794.

34. Emelianov A. V., Eremin A. V., Yatsenko P. I. The study of $\mathrm{C}_{2} \mathrm{~F}_{4} \mathrm{Br}_{2}$ dissociation kinetics using methods of atomic and molecular resonance absorption spectroscopy behind shock waves // J. Phys.: Conf. Ser. - 2018. - Vol. 946. - P. 012-070.

35. Bystrov N. S., Emelianov A. V., Eremin A. V., Yatsenko P. I. Direct measurements of rate coefficients for thermal decomposition of $\mathrm{CF}_{3} \mathrm{I}$ using shock-tube ARAS technique // J. Phys. D: Appl. Phys. - 2018. - Vol. 51 (18). - P. 184-204.

36. Javoy S., Mevel R., Paillard C. E. A study of $\mathrm{N}_{2} \mathrm{O}$ decomposition rate constant at high temperature: Application to the reduction of nitrous oxide by hydrogen // Int. J Chem. Kinet. - 2009. - Vol. 41. - P. 357-375.

37. Naudet V., Abid S., Paillard C.E. A High Temperature Chemical Kinetics Study of the $\mathrm{O}_{2}$ Dissociation and the O Atoms Recombination by ARAS // J. Chim. Phys. - 1999. - V. 96. - P. 1123-1145.

38. Thielen K., Roth P. Resonance absorption measurements of $\mathrm{N}$ and $\mathrm{O}$ atoms in high temperature NO dissociation and formation kinetics // Proc. Combust. Inst. - 1984. - Vol. 20. - P. 685-693.

39. Konnov A. A., De Ruyck J. Kinetic Modeling of Nitrogen Oxides Decomposition at Flame Temperatures // J. Combust Sci. Technol. - 1999. - Vol. 149. - P. 53-78.

40. Frassoldati A., Cuoci A., Faravelli T., Ranzi E. Kinetic Modeling of the Oxidation of Ethanol and Gasoline Surrogate Mixtures // Combust. Sci. Tech. - 2010. - Vol. 182. - P. 653-667.

41. Subith S. V., Sarathy S. M. On the High-Temperature Combustion of n-Butanol: Shock Tube Data and an Improved Kinetic Model // Fuel. - 2013. - Vol. 27 (11). - P. 7072-7080.

42. Bozzelli J.W., Chang A., Dean A.M. Analysis of the reactions $\mathrm{H}+\mathrm{N}_{2} \mathrm{O}$ and $\mathrm{NH}+\mathrm{NO}$ : pathways and rate constants over a wide range of temperature and pressure // Symp. Int. Combust. Proc. - 1994. Vol. 25. - P. 965-974.

43. Starikovskii A. Y. Kinetics and mechanism of reaction in $\mathrm{N}_{2} \mathrm{O}-\mathrm{CO}$ system at high temperatures // Khim. Fiz. - 1994. - Vol. - 13. - P. 94-120.

44. Roose T. R., Hanson R. K., Kruger C.H. Decomposition of NO in the Presence of $\mathrm{NH}_{3} / /$ Proc. Int. Symp. Shock Tubes Waves. - 1978. - Vol. 11. - P. 309-331.

45. Trenwith A. B. The kinetics of the oxidation of ethylene by nitrous oxide // J. Chem. Soc. - 1960. Vol. 79. - P. 3722-3726.

46. Allen M. T., Yetter R. A., Dryer F. L. The decomposition of nitrous oxide at $1.5<\mathrm{P}<10.5 \mathrm{~atm}$ and $1103<\mathrm{T}<1173$ K // Int. J. Chem. Kinet. V. 27 P. 883 - 909 Y. 1995 
47. Zuev A.P., Starikovskii A.Y. Reactions involving nitrogen oxides at high temperatures. The reaction of $\mathrm{N}_{2} \mathrm{O}$ with O // Khim. Fiz. - 1991. - Vol.10. - P. 179-189.

48. Kenwright R., Trenwith A.B. The kinetics of the oxidation of ethane by nitrous oxide. Part II. // J. Chem. Soc. - 1959. - Vol. 63. - P. 112-141.

49. Tomeczek J., Gradon B. The role of $\mathrm{N}_{2} \mathrm{O}$ and NNH in the formation of NO via HCN in hydrocarbon flames // Combust. Flame. - 2003. - Vol. 133. - P. 311-322.

50. Bauerle S., Klatt M., Wagner H. G. Investigation of the reaction of $\mathrm{CH}_{2}$ with $\mathrm{NO}$ at high temperatures // Ber. Bunsenges. Phys. Chem. - 1995. - Vol. 99. - P. 97-104.

51. Dagaut P., Lecomte F., Chevailler S., Cathonnet M. Experimental and Kinetic Modeling of Nitric Oxide Reduction by Acetylene in an Atmospheric Pressure Jet-Stirred Reactor // Fuel V.78 P. 1245 1252 Y. 1999

52. Chakraborty D., Lin M.C. Theoretical studies of methyleneamino $\left(\mathrm{CH}_{2} \mathrm{~N}\right)$ radical reactions. 1. Rate constants and product branching ratios for the $\mathrm{CH}_{2} \mathrm{~N}+\mathrm{N}_{2} \mathrm{O}$ process by ab initio molecular orbital/statistical theory calculations // J. Phys. Chem. A. - 1999. - Vol. 103. - P. 601-606.

53. Cohen N., Westberg K.R. Chemical kinetic data sheets for high-temperature reactions. Part II // J. Phys. Chem. Ref. Data. - 1991. - Vol. 20. - P. 1211-1311.

54. Hack W., Wagner H. G., Zaspypkin A. Elementary reactions of $\mathrm{NH}$ and $\mathrm{NH}\left(\mathrm{X}^{3} \Sigma\right)$ with $\mathrm{N}, \mathrm{O}$ and $\mathrm{NO} / /$ Ber. Bunsenges. Phys. Chem. - 1994. - Vol. 98. - P. 156-164.

55. McCullough R.W., Kruger C.H., Hanson R.K. A Flow Tube Reactor Study of Thermal Decomposition Rates of Nitric Oxide // Combust. Sci. Technol. - 1977. - Vol. 15. - P. 156-179.

56. Murrell J.N., Rodriguez J.A. Predicted rate constants for the exothermic reactions of ground state oxygen atoms and CH radicals // J. Mol. Struct. THEOCHEM. - 1986. - Vol. 139. - P. 354-376.

57. Tsuboi T., Hashimoto K. Shock Tube Study on Homogeneous Thermal Oxidation of Methanol // Combust. Flame. - 1981. - Vol. 42. - P. 265-291.

58. Peeters J., Boullart W., Devriendt K. CH $\left(\mathrm{a}^{4} \Sigma^{-}\right.$and/or $\left.\mathrm{X}^{2} \mathrm{II}\right)$ formation in the reaction between ketenyl radicals and oxygen atoms. Determination of the CH yield between 405 and $960 \mathrm{~K} / /$ J. Phys. Chem. 1995. - Vol. 99. - P. 3583-3591. 